EPJ Web of Conferences 48, 00015 (2013)

DOI: $10.1051 /$ epjconf/20134800015

(C) Owned by the authors, published by EDP Sciences, 2013

\title{
Irregular surfaces - measurements and ZEMAX simulations
}

\author{
Radek Melich ${ }^{\mathrm{a}}$, Pavel Psota, Vít Lédl, and Jan Václavík \\ Institute of Plasma Physics AS CR, v.v.i., TOPTEC, Sobotecká 1660, Turnov 51101, Czech Republic
}

\begin{abstract}
Surfaces produced on optical elements are loaded by different kinds of irregularities whose allowable amplitudes are prescribed by optical tolerances defined by an optical designer. An important feedback between realized and designed surfaces can be achieved using interferometry or profilometry measurements for instance. This article discusses different kinds of representation and visualization of those measurements in ZEMAX, enabling optical designer to evaluate an impact of the irregular surface on the optical system performance.
\end{abstract}

\section{Introduction}

In the nowdays optical industry more than before it is important to be able to measure the produced shape of the surface. Todays CNC machinery enables optical technicians to produce so precise surfaces that measurement techniques and precisions they reach are limiting factors as regards the final surface precision. However, a possibility to represent the measurement within an optical design software helps optical designers to investigate an impact of optical surfaces loaded by different kinds of irregularities on the entire optical system. This gives a change to more equally distribute the tolerance budget over the numerous surfaces and by that lose the tightest tolerances, so that final optical system is in a good balance.

For this reason a way for a data exchange between measuring devices and design software needs to be established. In the following sections that way is described as used in TOPTEC department of Institute of Plasma Physics AS CR, v.v.i.

\section{Interferometry and profilometry in TOPTEC}

Optical Centre TOPTEC is equiped with several kinds of interferometrical devices. First and most simple are two development plane interferometers of Fizeau type with possible measurement up to $200 \mathrm{~mm}$ diameter flats, respectively $350 \mathrm{~mm}$. These interferometers do not have any digital output but skilled optical technicians can easily and quickly used them in a production process for control and correction of polished flat surfaces.

Second type is a Twyman-Green spherointerferometer from FISBA company (today TRIOPTICS) which is equipped with several 4" objective of various $\mathrm{f} / \#$ to be able to measure different kinds of lenses. This interferometer is fully digitalized and enables measurement of surface distortion (among others) and its representation using Zernike Fringe coefficients or a pixel phase matrix.

a e-mail: melichr@ipp.cas.cz
Third and very unique type of interferometer, TOPTEC is equipped with, is 6" ASI interferometer (Aspherical Stitching Interferometer) from QED company. This interferometer based on Fizeau type uses advantages of a sub-aperture stitching and a variable null test equipment [1] to assure measurements up to $1000 \lambda$ departure from the best fit sphere. Also this interferometer can export measured data into Zernike Fringe coefficients or pixel phase matrix.

Another type of measurement in TOPTEC is profilometry. Instead of wavefront aberrations a direct deviation from the prescribed surface is measured. TOPTEC uses two kinds of profilometer devices in its laboratories. The first one is an optical non-contact profilometer which measurement technique is based on multi-wavelength interferometry [2]. This feature enable us to measure a distance with an accuracy of a nanometer within a range of up to $2 \mathrm{~mm}$ on a diameter up to $260 \mathrm{~mm}$. Measured data can be further represented in a form of pixel sag matrix.

Among other profilometer belongs Mytutoyo Legex 774 with its unique $3 \mathrm{D}$ measurement capability within a box of $710 \times 710 \times 460 \mathrm{~mm}$ with a precision of $0.35 \mu \mathrm{m}+$ $\mathrm{L} / 100 \mu \mathrm{m}$. Measured data can be also represented in a form of pixel sag matrix.

These measurement export capabilities give to an optical designer an excellent overview and feedback information on a performance of an optical system that is loaded by irregular surfaces.

\section{Irregular surfaces in ZEMAX}

Optical design software ZEMAX [3] disponees with several possible representations of irregular surfaces that describe surface distortion. In this paper we closely focus on the most used ones with respect to the irregularity measurement. These surfaces can be differentiated into four surface types with respect to way they are defined and what they describe. In ZEMAX they are called Grid Phase, Grid Sag, Zernike Fringe Phase and Zernike Fringe Sag.

This is an Open Access article distributed under the terms of the Creative Commons Attribution License 2.0, which permits unrestricted use, distribution, and reproduction in any medium, provided the original work is properly cited. 


\subsection{Grid Phase and Sag}

These surfaces can describe a phase of the wavefront traveling through the surface or they can describe an irregular sag of the surface. They have in common a way they are defined. To the ZEMAX software they are uploaded with imported ASCII file with a given structure. This structure is as follows:

nx ny delx dely unitflag xdec ydec $\mathrm{z} d z / d x d z / d y d 2 z / d x d y$ nodata

The very first line containing 7 values describes $\mathrm{nx} \times$ ny number of pixel in $\mathrm{x}$ and $\mathrm{y}$ directions. Values del $\mathrm{x}$ and dely define the increment in $x$ and $y$ directions. unitflag indicates the units of the data ( 0 for $\mathrm{mm}, 1$ for $\mathrm{cm}, 2$ for inch and 3 for meters). And if necessary it is possible to use $x d e c$ and ydec for decenter of the grid relative to the base surface. Further lines are data lines where $\mathrm{z}$ represents the phase, sag respectively. The first and second derivatives $\mathrm{dz} / \mathrm{dx}, \mathrm{dz} / \mathrm{dy}$ and $\mathrm{d} 2 \mathrm{z} / \mathrm{dxdy}$ are required for bicubic interpolation between data points. If all the derivatives are zero for every point in the file ZEMAX computes them automatically. More detailed description can be found in a ZEMAX manual [4].

Suggestion for using of Grid Phase surface is with interferometry data where the surface describe phase variations (wavefront error). For the direct surface deformations, as measured with profilometry for instance, the Grid Sag should be used instead.

Figure 1 shows the graphical representation of the given Grid Phase surface.

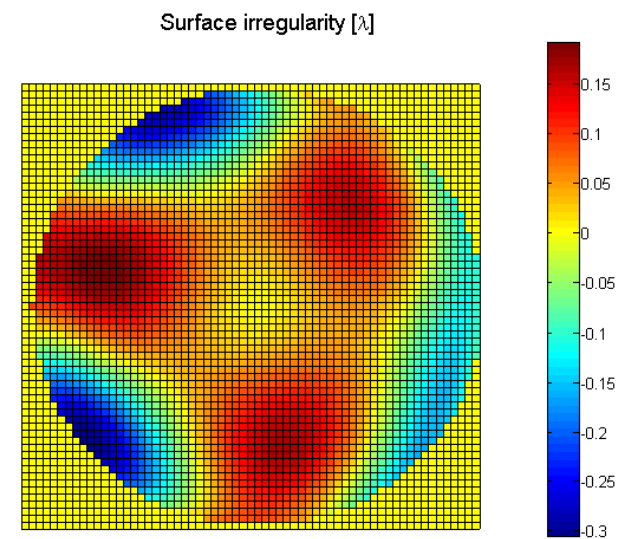

Fig. 1. Graphical representation of the Grid Phase surface. Values of individual pixels represent phase error on the surface in waves.

\subsection{Zernike Fringe Phase and Sag}

Next important representations of irregular surfaces in ZEMAX are Zernike Fringe Phase (ZFP) and Zernike Fringe
Sag (ZFS). They use 37 Zernike Fringe polynomials to define phase of the wavefront, respectively sag from the base surface. First 15 Zernike fringe polynomials, their mathematical expression, type of aberration and graphical expression, are shown in table 1.

Table 1. Mathematical representation of first 15 of 37 Zernike Fringe polynomials and their expression in a type of abberation and wavefront error.

\begin{tabular}{|c|c|c|c|c|}
\hline $\begin{array}{c}\text { ZERNIKE } \\
\text { TERM }\end{array}$ & $\begin{array}{c}\text { ZERNIKE } \\
\text { ORTHOGONAL } \\
\text { CIRCLE POLYNOMIAL }\end{array}$ & $\begin{array}{c}\text { RMS } \\
\text { ERROR }\end{array}$ & ABERRATION & $\begin{array}{l}\text { TYPE OF } \\
\text { WEAK MAP }\end{array}$ \\
\hline$Z_{0}$ & 1 & $Z_{0}$ & piston & \\
\hline$Z_{1}$ & $\rho \cos \theta$ & $\mathrm{Z} 1 / 2$ & \multirow{2}{*}{ distortion/tilt } & \\
\hline$Z_{2}$ & $\rho \sin \theta$ & $\mathrm{Z}=2 / 2$ & & \\
\hline$Z^{3}$ & $2 p^{2}-1$ & $Z_{3} / \sqrt{3}$ & $\begin{array}{l}\text { defocus/field } \\
\text { curvature }\end{array}$ & \\
\hline$Z_{4}$ & $\rho^{2} \cos 2 \theta$ & $Z_{4} / \sqrt{ } 6$ & \multirow{2}{*}{$\begin{array}{c}\text { primary } \\
\text { astigmatism }\end{array}$} & \\
\hline$Z s$ & $\rho^{2} \sin 2 \theta$ & $Z s / \sqrt{ } 6$ & & \\
\hline$Z_{6}$ & $\left(3 p^{3}-2 p\right) \cos \theta$ & $Z_{6} / \sqrt{ } 8$ & \multirow{2}{*}{ primary coma } & \\
\hline $\mathrm{Z}^{7}$ & $\left(3 p^{3}-2 p\right) \sin \theta$ & $\mathrm{Z}>/ \sqrt{ } 8$ & & \\
\hline$Z_{a}$ & $6 p^{4}-6 p^{2}+1$ & $\mathrm{Za} / \sqrt{5}$ & $\begin{array}{l}\text { primary } \\
\text { spherical } \\
\text { aberration }\end{array}$ & \\
\hline$Z 9$ & $\rho^{3} \cos 3 \theta$ & $Z 9 / \sqrt{ } 8$ & elliptical coma & \\
\hline$Z^{10}$ & $\rho^{3} \sin 3 \theta$ & $\mathrm{Z}_{10} / \sqrt{8}$ & $\begin{array}{c}\text { (ARROWS, } \\
\text { TREFOIL) }\end{array}$ & \\
\hline$Z_{11}$ & $\left(4 p^{4}-3 p^{2}\right) \cos 2 \theta$ & $Z_{11} / \sqrt{ } 10$ & secondary & \\
\hline$Z_{12}$ & $\left(4 p^{4}-3 p^{2}\right) \sin 2 \theta$ & $Z_{12} / \sqrt{ } 10$ & astigmatism & \\
\hline$Z_{13}$ & $\left(10 \rho^{5}-12 \rho^{3}+3 \rho\right) \cos \theta$ & $Z_{13} / \sqrt{ } 12$ & \multirow{2}{*}{ seœndary coma } & \\
\hline$Z_{14}$ & $\left(10 p^{5}-12 p^{3}+3 \rho\right) \sin \theta$ & $Z_{14} / \sqrt{12}$ & & \\
\hline$Z^{15}$ & $20 p^{6}-30 p^{4}+12 p^{2}-1$ & $Z_{15} / \sqrt{7}$ & $\begin{array}{l}\text { secondary } \\
\text { spherical } \\
\text { aberration }\end{array}$ & \\
\hline
\end{tabular}

Basis for ZFP is a standard surface that support plane, sphere and conic surface plus an additional phase term $\Phi$ defined in a form

$$
\Phi=M \sum_{i=1}^{N} 2 \pi A_{i} Z_{i}(\rho, \varphi) .
$$

Here $N$ stands for the number of Zernike coefficients, $A_{i}$ (units of waves) is the coefficient on the $i^{\text {th }}$ Zernike Fringe polynomial, $\rho$ is the normalized radial ray coordinate, $\varphi$ is the angular ray coordinate and $M$ is the diffraction order.

Basis for ZFS is an even asphere which support planes, spheres, conics and polynomial aspheres plus an additional aspheric surface term defined with Zernike Fringe polynomials. The final sag is then in a form of

$$
z=\frac{c r^{2}}{1+\sqrt{1-(1+k) c^{2} r^{2}}}+\sum_{i=1}^{8} \alpha_{i} r^{2 i}+\sum_{i=1}^{N} A_{i} Z_{i}(\rho, \varphi)
$$

where $c$ stands for the curvature of the surface, $r$ is the radial ray coordinate, $k$ is the conic constant, $\alpha_{i}$ are coefficients of the polynomial asphere, $A_{i}$ are coefficients of 
Zernike Fringe polynomials $Z_{i}, \rho$ is the normalized radial ray coordinate and $\varphi$ is the angular ray coordinate.

Using Zernike Fringe polynomials notation the irregularity of a surface can be mathematically expressed. Table 2 shows different spacial frequencies of wavefront error - from low to high frequencies - using different set of Zernike Fringe coefficients.

Table 2. Different spacial frequencies of wavefront error - from low to high frequencies - using different set of Zernike Fringe coefficients.

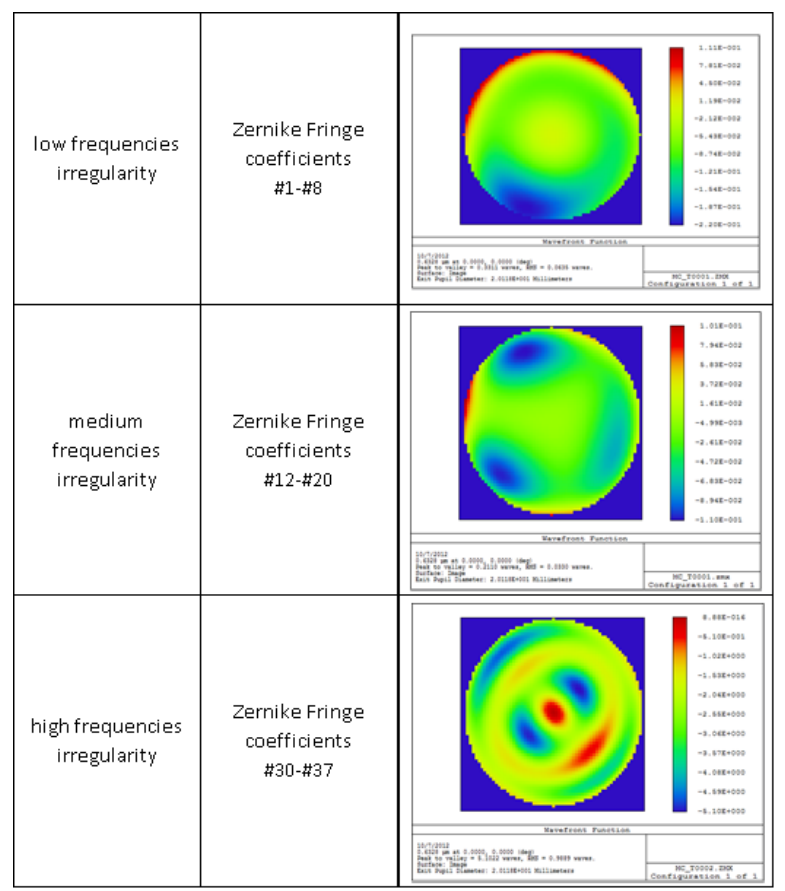

ZFP is suggest to use with interferometry data which describe phase variations (wavefront error). For the direct surface deformations, as measured with profilometry for instance, ZFS should be used instead.

\subsection{Other irregular ZEMAX surfaces}

ZEMAX offers also another surfaces that can be used to expressed interferometric data. Among them belongs Zernike Standard Phase and Zernike Standard Sag. They are similar to ZFP and ZFS but they use up to 231 Zernike Standard polynomials to define their surface. Compare to Fringe polynomials they can be more detailed and precise in the expense of computational time.

Another expression - Zernike Annular Phase - uses up to 231 Zernike annular polynomials to define surface phase. These types of polynomials are often used in astronomy where the pupil has an annular shape.

\section{Tolerance analysis example}

Irregular surfaces of individual optical elements within an optical system influence its final performance. Mechan- ical errors or simple deviations from design values (like decentering, shift, tilt or radius deviation) has usually stronger impact. However, these errors can be more easily controlled and eliminated and therefore the surface irregularities became indispensable.

In the following we provide a tolerance study of $\mathrm{F} / 5$ doublet with respect to the following possible aberrations: decentering (both surfaces and elements), shift (both surfaces and elements), tilt (both surfaces and elements), radius deviation and surface irregularity. As a compensator we have chosen a back focal length with margins of $+/-1$ mm.

The doublet has a prescription as stated in the table 3 . This doublet is designed with respect to the best of transmission RMS wavefront error at $633 \mathrm{~nm}$ for on-axis beam. With this design we achieve $\lambda / 40$ RMS transmission wavefront error. Of course this is an ideal state and wavefront will degrade as we start to take in account mentioned individual production aberrations.

Table 3. Prescription of designed doublet optimized for on-axis RMS wavefront aberration at $633 \mathrm{~nm}$.

\begin{tabular}{|c|c|c|c|c|c|}
\hline Lens ID & RoC [mm] & $\begin{array}{c}\text { Thickness } \\
{[\mathrm{mm}]}\end{array}$ & Glass & $\begin{array}{c}\text { CA/Diameter } \\
{[\mathrm{mm}]}\end{array}$ & $\begin{array}{c}\text { Distance } \\
\text { between } \\
\text { lenses [mm] }\end{array}$ \\
\hline \multirow{2}{*}{ L1 } & 392.00 & 25.00 & BK7 & $145 / 152$ & \multirow{2}{*}{0.5} \\
\cline { 2 - 5 } & -1200.00 & 20.00 & SF2 & $145 / 152$ & \\
\cline { 1 - 2 } & -584.50 & -924.85 & \multirow{2}{*}{20.52} & \\
\cline { 2 - 5 }
\end{tabular}

The table 4 shows an impact of reasonable chosen errors $(10 \mu \mathrm{m}$ for decentering, $0.1 \mathrm{~mm}$ for shifts, 1 arc seconds for tilts and $\lambda / 5$ for surface irregularities) and their impact on the final doublet performance. Within a chosen margin error it can be achieved $\lambda / 8$ RMS wavefront error, which was also proved by Monte-Carlo analysis that combine all deviations (see figures 2 and 3 ).

Results of tolerance analysis shows that worst offenders are mainly the tilts - of both surface and element see table 4 and impact of these errors on nominal criterion (RMS wavefront aberration). On the same level it is the rest of tested aberrations. From this simulation we can see that chosen surface irregularities margins can be about two-time more relaxed and still achieve reasonable $\lambda / 8$ wavefront error performance.

\section{Conclusion}

The paper describes different types of interferometry and profilometry measurements, that TOPTEC is equipped with, and their possible measurements representation within the optical design software ZEMAX. This data flow chain enables an optical designer to decide about the final impact of selected surface irregularity onto an optical system performance.

This work was supported by the European Regional Development Fund and the Ministry of Education, Youth and Sports of the Czech Republic in the 


\section{EPJ Web of Conferences}

Table 4. Production errors and their impact on nominal criterion (RMS wavefront aberration).

\begin{tabular}{|c|c|c|c|c|c|c|}
\hline Type & surfaces & surfaces & units & Value & Criterion & $\begin{array}{c}\text { Change to nominal } \\
\text { criterion }\end{array}$ \\
\hline surface tilt & 3 & & arcmin & 1 & 0.088 & 0.080 \\
\hline surface tilt & 3 & & arcmin & -1 & 0.088 & 0.080 \\
\hline surface tilt & 4 & & arcmin & -1 & 0.056 & 0.043 \\
\hline surface tilt & 4 & & arcmin & 1 & 0.056 & 0.043 \\
\hline element tilt & 3 & 4 & arcmin & -1 & 0.051 & 0.036 \\
\hline element tilt & 3 & 4 & arcmin & 1 & 0.051 & 0.036 \\
\hline surface radius & 3 & & fringe & 4 & 0.050 & 0.034 \\
\hline surface irregularities & 3 & & fringe & 0.2 & 0.049 & 0.033 \\
\hline surface irregularities & 4 & & fringe & -0.2 & 0.048 & 0.032 \\
\hline layer thickness & 2 & 4 & $\mathrm{~mm}$ & 0.1 & 0.047 & 0.030 \\
\hline surface irregularities & 1 & & fringe & 0.2 & 0.047 & 0.029 \\
\hline surface irregularities & 2 & & fringe & -0.2 & 0.046 & 0.029 \\
\hline surface radius & 4 & & fringe & -4 & 0.044 & 0.024 \\
\hline surface tilt & 2 & & arcmin & 1 & 0.043 & 0.023 \\
\hline surface tilt & 2 & & arcmin & -1 & 0.043 & 0.023 \\
\hline layer thickness & 1 & 2 & $\mathrm{~mm}$ & -0.1 & 0.042 & 0.020 \\
\hline surface radius & 1 & & fringe & 4 & 0.041 & 0.019 \\
\hline surface radius & 2 & & fringe & -4 & 0.039 & 0.014 \\
\hline layer thickness & 3 & 4 & $\mathrm{~mm}$ & -0.1 & 0.037 & 0.009 \\
\hline surface decenter & 3 & & $\mathrm{~mm}$ & 0.01 & 0.037 & 0.004 \\
\hline surface decenter & 3 & & $\mathrm{~mm}$ & -0.01 & 0.037 & 0.004 \\
\hline element decenter & 3 & 4 & $\mathrm{~mm}$ & -0.01 & 0.036 & 0.003 \\
\hline element decenter & 3 & 4 & $\mathrm{~mm}$ & 0.01 & 0.036 & 0.003 \\
\hline surface decenter & 4 & & $\mathrm{~mm}$ & 0.01 & 0.036 & 0.001 \\
\hline surface decenter & 4 & & $\mathrm{~mm}$ & -0.01 & 0.036 & 0.001 \\
\hline surface decenter & 2 & & $\mathrm{~mm}$ & 0.01 & 0.036 & 0.000 \\
\hline surface decenter & 2 & & $\mathrm{~mm}$ & -0.01 & 0.036 & 0.000 \\
\hline element decenter & 1 & 2 & $\mathrm{~mm}$ & 0 & 0.036 & 0.000 \\
\hline element decenter & 1 & 2 & $\mathrm{~mm}$ & 0 & 0.036 & 0.000 \\
\hline element tilt & 1 & 2 & arcmin & 0 & 0.036 & 0.000 \\
\hline
\end{tabular}
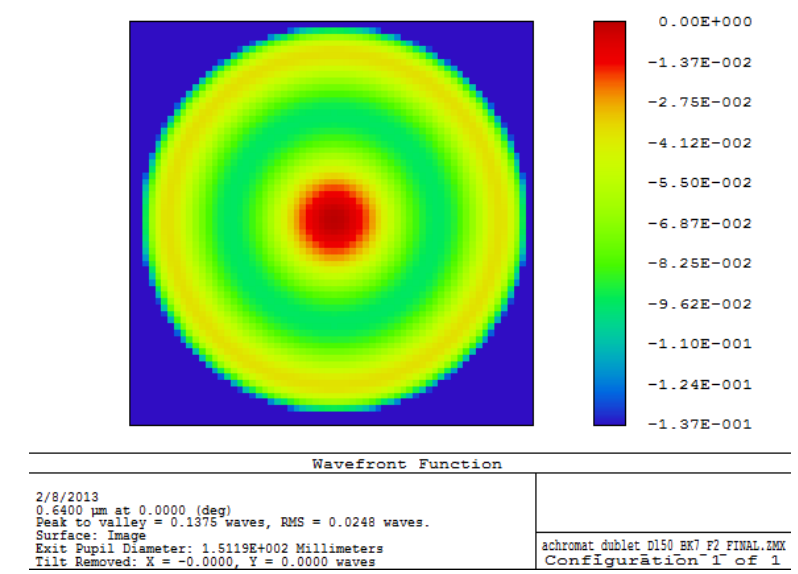

$0.00 E+000$

Fig. 2. The on-axis RMS wavefront map of an ideal doublet. It reaches values of $\lambda / 40$.
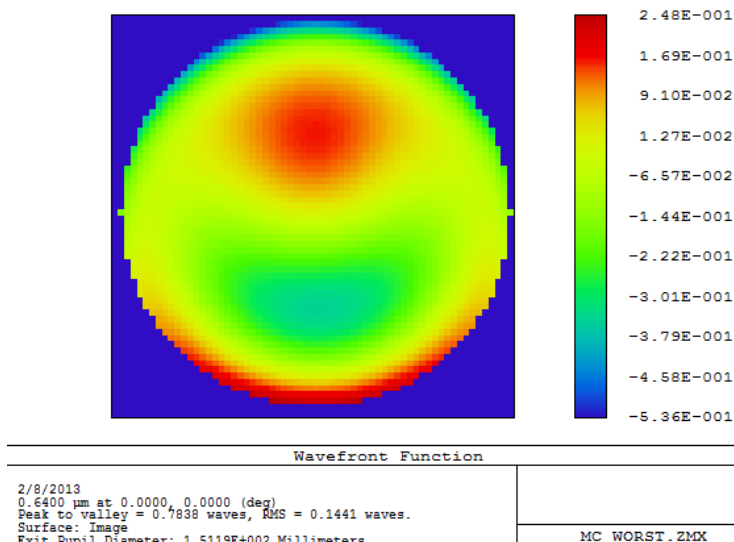

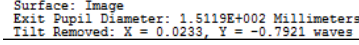

Fig. 3. Worst case $10000 \mathrm{MC}$ simulation of the doublet loaded by proposed aberrations. The figure shows the on-axis RMS wavefront of an ideal doublet. It reaches values of $\lambda / 8$

Project No. CZ.1.05/2.1.00/03.0079: Research Center for Special Optics and Optoelectronic Systems (TOPTEC).

\section{References}

1. Andrew Kulawiec, Laser Focus World, 46, (2010)

2. www.luphos.de

3. www.radiantzemax.com

4. Radiant ZEMAX LCC, ZEMAX 12 - User's manual, 335-338 (2012) 